\title{
AN AMATEUR'S EXPERIENCES WITH BYBLIS GERMINATION
}

\section{HAWKEN CARLTON・Colorado•USA・hawken.carlton5@gmail.com}

Most carnivorous plant growers who've been in the game for at least a year or two tend to be passingly familiar with at least one of the several Byblis species currently described. Byblis liniflora in particular is a common weed, easy to germinate and easy to grow. The rest of the genus, however, presents a series of challenges to successful cultivation (and subsequent maturation), the first of which being successful germination of the seeds.

While $B$. liniflora often requires little more than a quick soak in water and then sowing on moist soil to germinate (though in this method it may take several weeks), the rest of the genus possesses distinctly fire-adapted seeds which require some form of special treatment in order to germinate. Among the options that cultivators have come up with: sowing on moist soils and lighting cool grass fires over the top, before pouring water into the ashes; treating with commercial smoke disks or "liquid smoke" applications for similar chemicals; soaking seeds in an approximately $10 \%$ bleach solution; and, perhaps most commonly now, treating seeds with a gibberellic acid (GA3) solution. In previous years I personally have had limited access to smoke products or viable GA3 (eBay sources are not always reliable for quality ingredients), and so have attempted several other options such as the grass fire (or variations thereof) and bleach treatments, settling mainly on the latter due to somewhat higher success.

More recently, however, I managed to acquire both viable GA3 powder was well as a number of Byblis species I had not had access to before, and saw an opportunity to conduct an experiment of my own on which method (bleach or GA3) is the most viable, particularly for a novice grower who may not readily have access to working chemicals.

\section{Bleach Treatment}

As bleach is a highly caustic chemical it is very important that this method is performed with care and hands are washed thoroughly after use. A proper solution for treatment is a dilution of approximately $10 \%$ household bleach to $90 \%$ pure water. Seeds should be placed in the solution and fully submerged with all sides exposed to the solution, and then carefully monitored. The bleach will remove a fair portion of the outer seed coat and the inhibitive chemicals held in it over a period of several minutes, turning the normally black seeds to a lighter shade of purple, brown, or gray; if you watch the seeds you will see a black or purplish trail or cloud seep out from the seed as it soaks. Seeds should be removed from the solution and rinsed as soon as a change in color becomes apparent; if they are left long enough to turn light gray or white, the whole seed coat has been removed or the bleach has penetrated all the way through and the embryo will be compromised.

Once fully rinsed, the seeds can then be sown in a pot on the proper soil (I personally use a sandy peat-based mix, sometimes topped with a very thin layer of milled sphagnum to help ward off mold and damping off), and germination of viable seeds should occur within 1-2 weeks.

\section{GA3 Treatment}

Treating seeds with GA3 is somewhat less touchy than the bleach method, and if spilled on the hands is less of a concern (though washing is still advised). Byblis seeds should be soaked in a concentration of approximately $1000 \mathrm{ppm}$, which can be achieved by dissolving approximately 100 


\begin{tabular}{|l|c|c|}
\hline \multicolumn{2}{|c|}{ Table 1. Germination of Byblis seed (number germinated of number sown). } \\
\hline Species & Bleach Method & GA3 Method \\
\hline B. filifolia "Pago Giant" & 3 of 6 & 10 of 12 \\
\hline B. filifolia "Hidden Valley" & 3 of 5 & 5 of 5 \\
\hline B. rorida "Lake Campion" & 2 of 3 & 2 of 3 \\
\hline B. guehoi & 3 of 5 & 5 of 5 \\
\hline B. aquatica "Robust Form" & 1 of 5 & 3 of 5 \\
\hline B. lamellata $\times$ gigantea & 0 of 4 & 4 of 4 \\
\hline
\end{tabular}

mg of GA3 powder in $100 \mathrm{ml}$ of water. Warm water is best, and full dissolution can be achieved by allowing the solution to sit overnight; dissolving the powder in a very small amount of warm ethanol or rubbing alcohol can also speed up the dilution process. Once the solution is prepared and all powder is visibly dissolved, annual Byblis species can be soaked for 24 hours before sowing, and the perennial species for 5-6 days. Then, seeds can be placed in pots in a similar regimen as in the bleach treatment. Germination should similarly occur anywhere between 4 days and about 3 weeks after.

\section{Germination Results}

The species/forms (Table 1) each had one group of seeds treated with bleach and one with GA3, with at least 3 seeds in each group (up to 12 seeds per group, determined by the number of seeds I had to work with for each).

Out of these, all test groups tended to have the first germination of seeds within 10 days of sowing, continuing up to approximately 4 weeks after sowing, so time until germination differed little between the two methods. This is where the similarities end, however.

For all but B. rorida "Lake Campion" (likely primarily due to the small number of seeds I had to work with for that form, only about 3 seeds per test group), GA3 treatment resulted in a higher germination rate (Table 1). Additionally, at first glance the GA3 germinated seedlings also appeared to be more robust than those germinated via the bleach method, and continued to be so as they've matured.

The B. filifolia "Pago Giant" plants from the bleach treatment that have now reached flowering stage have failed to produce pollen, while plants blooming from the GA3 treatment readily set pollen. Byblis filifolia "Hidden Valley" plants from the bleach treatment tend to be highly sensitive to leaf disturbance, with bumped or bent (even occasionally fed) leaves often wilting for no other apparent reason, but GA3 treated plants have shown no such response. Byblis guehoi from the bleach treatment appear to grow lankier, as scrambling vines rather than the bushy species they are typically known as, while GA3 seedlings are developing far more robustly and with denser growth (this may, however, be in part due to differences in the seed source material, and more testing will need to be done to determine if it is in fact the seed treatment or not that is resulting in this odd growth form). In B. aquatica, while the smallest plant is among those germinated from GA3, all plants from the GA3 treatment are flowering far more heavily than the singular plant that germinated from the bleach method. For the perennial species used, of course, no comparison can be made; bleach does not appear to be a viable option for germinating those species.

Figure 1 (left panel) shows representative photos that I was able to take of the germinating seedlings; Figure 1 (right panel) is of a selection of the flowering adult plants that have resulted. 

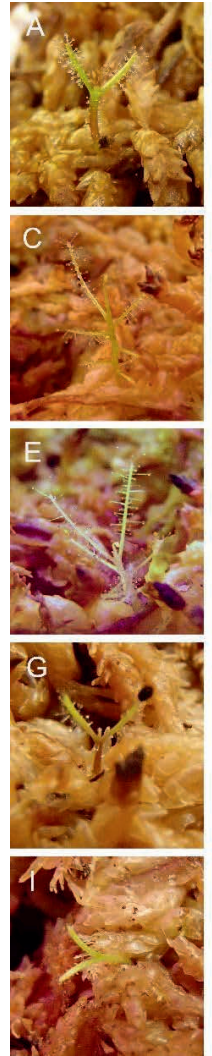
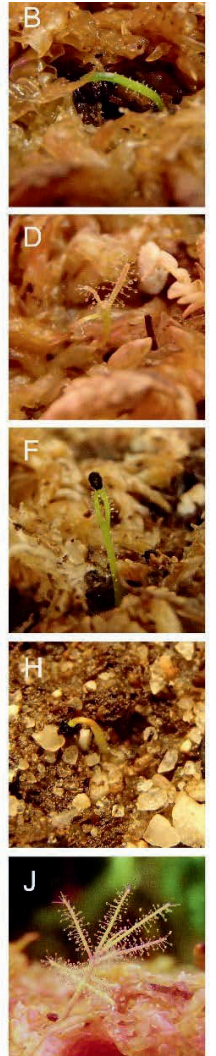
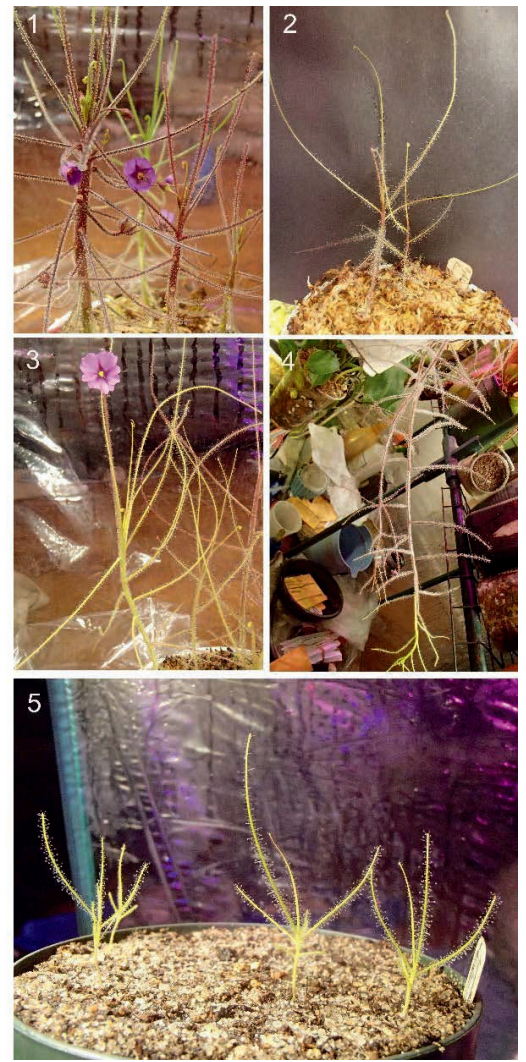

Figure 1: Left panel: A B. aquatica bleach treatment; B B. aquatica GA3 treatment; C B. filifolia "Hidden Valley" bleach; D B. filifolia "Hidden Valley" GA3; E B. filifolia "Pago Giant" bleach; F B. filifolia "Pago Giant" GA3; G B. guehoi GA3; H B. lamellata x gigantea GA3; I B. rorida "Lake Campion" GA3; J B. rorida "Lake Campion" bleach. Right panel: 1 B. aquatica "Robust Form" with GA3 plants in front and bleach plant in back; 2 B. filifolia "Hidden Valley" bleach plants; 3 B. filifolia "Pago Giant" bleach plant in bloom, but failing to produce pollen; 4 B. guehoi bleach plants demonstrating their lanky growth habit; 5 B. lamellata $\times$ gigantea GA3 seedlings.

From the preliminary results I've acquired from this, I am drawing the conclusion that despite ease of access, germination of Byblis seeds with bleach treatment is a far inferior method to GA3 (which is, luckily, becoming more readily available from more reliable sources). Further experimentation, particularly with the next generations of plants and hopefully other localities or species, is necessary for more definitive conclusions.

Among data already gathered as well but unable to be compared as only one treatment was used for each variety: $B$. gigantea germinates from 5-6 days of GA3 treatment similar to the $B$. lamellata $\times$ gigantea hybrid; $B$. rorida "Taylor's Lagoon" successfully germinated from bleach treatment, but had poor overall germination rates; $B$. liniflora, while not technically needing any particular treatment, germinates at extremely high success and very rapidly after being give a 24-hour GA3 soak. 